\title{
SEPARATION OF PLASTIC PARTICLES IN ELECTROSTATIC FIELD PRODUCED BY ELECTRODES OF OPTIMIZED SHAPE
}

Shape optimization of electrodes for the device for electrostatic separation of triboelectrically charged plastic particles is carried out. The objective function maximizes the efficiency of separation consisting in the highest possible number of particles falling down to the prescribed bins. Electric field in the system is solved numerically, using the fully adaptive higher-order finite element method. The movement of particles in the device influenced by the Coulomb force is determined by means of an adaptive Runge-Kutta-Fehlberg method with a time varying time step. The shape optimization is carried out using a technique based on genetic algorithms. The methodology is illustrated by an example whose results are discussed.

Keywords: Charged plastic particles, triboelectric effect, higher-order finite element method, genetic algorithms, adaptivity.

\section{Introduction}

Nowadays, an intensive research is conducted worldwide, aimed at the possibilities of recycling plastic materials, because in a lot of applications it may well replace the new ones. The necessary prerequisite of this reprocessing is a high-quality separation of particular kinds of plastics preliminarily ground into small sphereshaped particles. One of the advanced techniques of separation of these particles is based on the triboelectric effect [1-3]. It is known that when electrically non-conducting particles of two different levels come into contact with electric charge, one of them becomes more positive (or negative) with respect to another one. And when such charged particles get into electric field, their movement is then mainly affected by the charge that they carry.

More accurately, the trajectories of the charged particles moving in electric field are influenced by the Coulomb force exerted on it by this field, gravity and drag aerodynamic forces. And these trajectories predetermine their impact points, in other words, the places where they fall down. It is clear from Fig. 1 showing a typical separator of this kind.

The separator consists of two electrodes, one of them being grounded. The voltage of the other electrode is used to be on the order of tens $\mathrm{kV}$. The electrodes may be covered by Teflon or another insulating material that prevents recharging of the particles in case of the direct impact with them. The mixture of charged particles is delivered by the feeder. At the bottom of the device there are several recycle bins used for accumulating of particular levels of plastics. One of the principal demands is to tune the shape of the electrodes and widths of the bins so that the particles of different levels should fall down exactly to the corresponding bin.

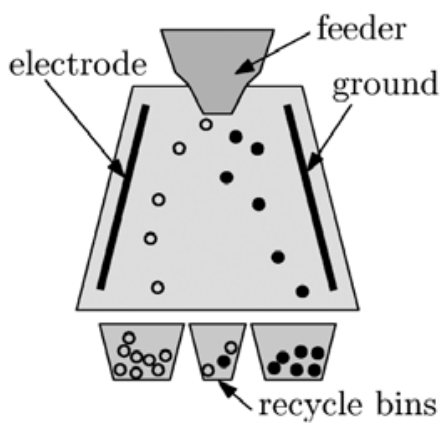

Fig. 1 Scheme of a typical separator

The problems of mapping electric field within the separator and trajectories of particles of plastics were dealt with by several authors (see, for example, [4-5]). Their approach, however, was based on low-order mapping techniques. The problem of the shape optimization of both electrodes (and also bins) was solved rather by a comparison of several different arrangements, without applying direct optimization techniques.

The paper represents an organic continuation of recent study [6] aimed at the shape optimization of the electrodes that was carried out using the conjugate gradient method. With respect to the discontinuousness of both functionals the method turned out to be rather disadvantageous for this application. Based on the results described in this paper we changed the formulation of the task in the sense of an appropriate change of both functionals and description of the shapes of the electrodes.

\footnotetext{
* Frantisek Mach ${ }^{1}$, Pavel Kus ${ }^{1}$, Pavel Karban ${ }^{1}$, Ivo Dolezel ${ }^{2}$

${ }^{1}$ Faculty of Electrical Engineering, University of West Bohemia, Pilsen, Czech Republic, E-mail: fmach@kte.zcu.cz

${ }^{2}$ Czech Technical University, Faculty of Electrical Engineering, Prague, Czech Republic
} 


\section{Formulation of the problem}

Let us start with a possible basic arrangement depicted in Fig. 2 (its principal dimensions being given in $\mathrm{mm}$ ). The particles of plastics of charge $Q$ and initial velocity $v_{0}$ get to the space between two electrodes, one of them being grounded. There they are deflected according to their charge and fall down into the recycle bins (presently, we neglect the Coulomb forces acting among the particles, so that their movement is driven mainly by the external electric field). The first task is to find their trajectories and evaluate the effectiveness of separation in this basic arrangement.

The second step is the optimization of the shape of the electrodes. Their basic shape is characterized by an efficiency defined as a ratio of particles of the given type that fall down to the corresponding bin versus the total number of particles of this kind And the aim of this optimization is to increase this ratio on the highest value possible.

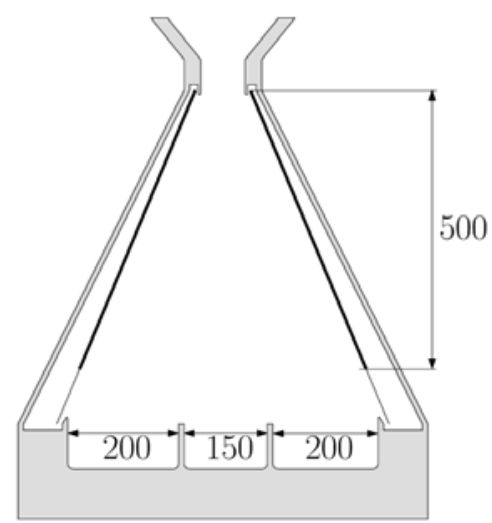

Fig. 2 Basic scheme of the separator (dimensions in $\mathrm{mm}$ )

\section{Continuous mathematical model}

Electric field in the working space of the separator is described by the equation for the electric potential $\varphi$ (see [7-8])

$$
\operatorname{div}(\varepsilon \operatorname{grad} \varphi)=0,
$$

where $\varepsilon$ is the dielectric permittivity. The boundary conditions are given by the known values of the electric potential on the electrodes and the Neumann condition along the artificial boundary placed at a sufficiently distance from the device.

The movement of the particle obeys the equations for its velocity $v$ and trajectory $s$ in the forms

$$
m \frac{d v}{d t}=F_{e}+F_{a}+F_{g}, \quad v=\frac{d s}{d t},
$$

where $F_{e}$ is the Coulomb force acting on the particle. This force is given by the relation

$$
F_{e}=Q E=-\operatorname{grad} \varphi,
$$

where $E$ denotes the local value of the electric field strength.

Symbol $F_{a}$ represents the aerodynamic resistance that is given by the formula

$$
F_{a}=-v \frac{1}{2} \rho c S v
$$

$c$ being the friction coefficient (depending on geometry of the particle), $\rho$ denotes the density of ambient air, $S$ is the characteristic surface of the particle and $v$ stands for the module of its velocity. Finally,

$$
F_{g}=m g \text {, }
$$

where $m$ denotes the mass of the particle and $g$ is the gravitational acceleration.

The corresponding initial conditions read

$$
v(0)=v_{0}, s(0)=s_{0}
$$

where $s_{0}$ is the entry position of the particle in the separator. Equation (2) is strongly nonlinear due to the first and second terms on the right-hand side.

\section{Numerical solution}

The above model (equations (1) and (2)) was solved numerically. For the field computations (1) we used our own code Agros2D [9], which is a powerful user's interface serving for pre-processing and post-processing of the problems solved. The code collaborates with the library Hermes [10] containing the most advanced algorithms for a fully adaptive solution of systems of generally nonlinear and nonstationary second-order partial differential equations (PDEs) based on the finite element method of higher order of accuracy.

Both codes written in $\mathrm{C}^{++}$are freely distributable under the GNU General Public License. The most important and in some cases quite unique features of the codes follow:

- Solution of the system of PDEs is carried out monolithically, which means that the resultant numerical scheme is characterized by just one stiffness matrix. The PDEs are first rewritten into the weak forms whose numerical integration provides its coefficients. The integration is performed using the Gauss quadrature formulas.

- Fully automatic $h p$-adaptivity. When adaptivity is required, in every iteration step the solution is compared with the reference solution (realized on an approximately twice finer mesh), and the distribution of error is then used for selection of candidates for adaptivity. Based on sophisticated and subtle algorithms the adaptivity is realized either by a subdivision of the candidate element ( $h$-adaptivity), by its description by a polynomial of a higher 
order ( $p$-adaptivity), or by a combination of both above options ( $h p$-adaptivity).

- Each physical field can be solved on quite a different mesh that best corresponds to its particulars. This is of great importance, for instance, for respecting skin effect in the magnetic field, while the temperature field is usually smooth. Special powerful higher-order techniques of mapping are then used to avoid any numerical errors in the process of assembly of the stiffness matrix.

- In nonstationary processes every mesh can change in time, in accordance with the real evolution of the corresponding physical quantities.

- Easy treatment of the hanging nodes appearing on the boundaries of subdomains whose elements have to be refined. Usually, these nodes bring about a considerable increase of the number of the degrees of freedom (DOFs). The code contains higherorder algorithms for respecting these nodes without any need of an additional refinement of the external parts neighboring with the refined subdomain.

- Curved elements able to replace curvilinear parts of any boundary by a system of circular or elliptic arcs. These elements mostly allow reaching highly accurate results near the curvilinear boundaries with very low numbers of the DOFs.

The movement of the particles affected by electric field, gravity and drag aerodynamic resistance is modeled by an adaptive RungeKutta-Fehlberg method with a time-varying time step.

\section{Illustrative example}

Two kinds of particles were considered for the computations: polyethylentereftalate (PET) with positive charges and polyvinylchloride (PVC) with negative charges. Their charges and dimensions obey the normal distribution with parameters in Table 1. Their numbers $n=250$ for each kind of material. The voltage between the electrodes was $U=30 \mathrm{kV}$.

Selected parameters of the particles

Table 1

\begin{tabular}{|c|c|c|c|c|c|}
\hline \multirow{2}{*}{ Type } & Density & \multicolumn{2}{|c|}{ Radius } & \multicolumn{2}{c|}{ Charge } \\
\cline { 2 - 6 } & $\left(\rho \mathrm{kg} / \mathrm{m}^{3}\right)$ & $\mu(\mathrm{mm})$ & $\sigma(\mathrm{mm})$ & $\mu(\mathrm{C})$ & $\sigma(\mathrm{C})$ \\
\hline PET & 1330 & 2 & 0.25 & $+0.25 \mathrm{E}-9$ & $0.8 \mathrm{E}-10$ \\
\hline PVC & 1370 & 2 & 0.25 & $-0.5 \mathrm{E}-9$ & $0.8 \mathrm{E}-10$ \\
\hline
\end{tabular}

Symbol $\mu$ denotes the median and $\sigma$ is the variance.

For example, Figs. 3, 4 and 5 show the distributions of the radii and charges of the PET and PVC particles.

As the particles enter the separator in different places between the electrodes, Fig. 6 shows the distribution of their initial positions.

The goal of this work is not only to calculate the electric field in the device and use it to find a trajectory of the particles, but also

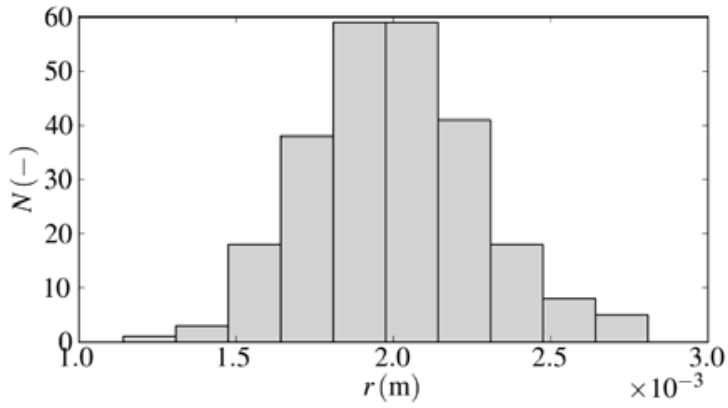

Fig. 3 Distribution of radii of the PET and PVC particles

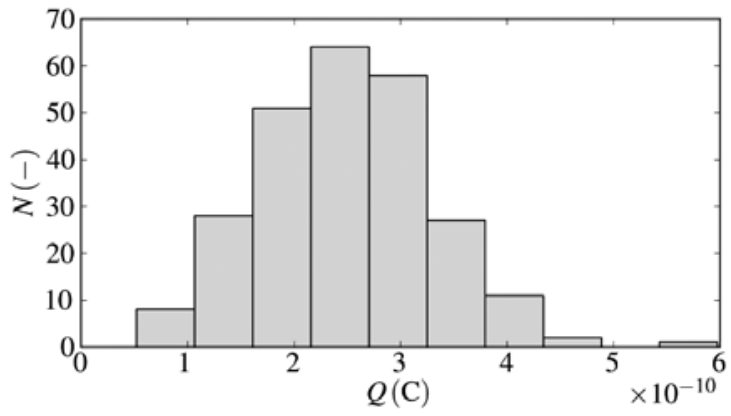

Fig. 4 Distribution of charges of the PET particles

to find such a design of the electrodes that the number of wellplaced particles is maximized. To achieve this, both electrodes are divided into several segments. By changing relative angles of the neighboring segments, different shapes of electrodes may be obtained. The objective functional of the optimization is the ratio of correctly placed particles, which should be maximized. According to a discrete nature of the objective functional, it is not possible to use any gradient-based optimization algorithm, which might be the first choice otherwise. We used approach based on genetic algorithms instead. The vector containing angles of segments serves as a "genome". An initial population of such genomes representing different designs is created. Then the operators of mutation and crossover are applied and designs with highest values of the objective functional are selected to the next generation. After sufficient number of iterations, the best design is selected as a product of the optimization.

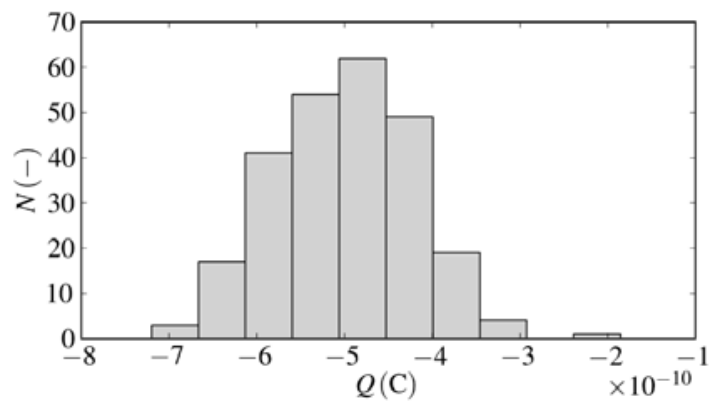

Fig. 5 Distribution of charges of the PVC particles 


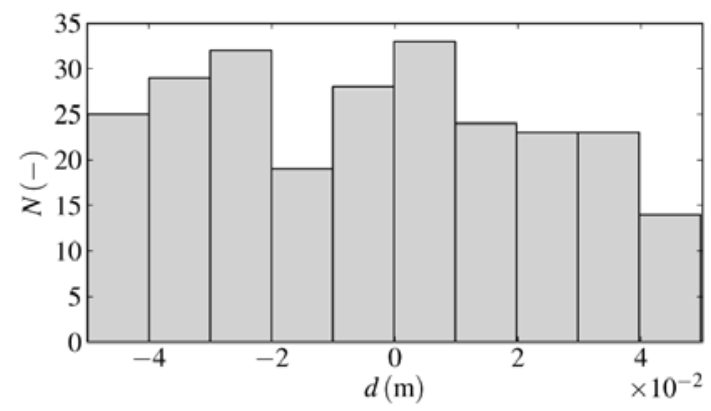

Fig. 6 Distribution of the initial positions of particular particles

More specifically, we tested two different functionals $F$ and $G$ with thirteen variable parameters. Every electrode was divided into four segments (three of them forming the electrode itself, the fourth one without voltage representing a barrier, see Fig. 7).

The border points are denoted by letters $A_{1}, B_{1}, C_{1}, A_{2}, B_{2}$ and $C_{2}$. These points can move in both directions within a prescribed range, which represents twelve variable parameters. The last parameter defines the place of the central bin and is given by the distance of the center of this bin from the center of the separator

The functional $F$ describes how many particles fall into the correct bin, while the functional gives the sum of the average distances of the PET and PVC particles from the desired points (centers) of the corresponding bins.

The convergence of the algorithm was in every step evaluated from the best results obtained for both functionals $\left(F_{\max }, G_{\min }\right)$. Its evolution is shown in Fig. 8. We can see there seemingly steady states (for example, in case of $F_{\max }$ between the sixth and tenth populations) caused by the effort of the algorithm to fill in the vacancies in the Pareto front. About 22 populations seem to be enough for obtaining the steady state.

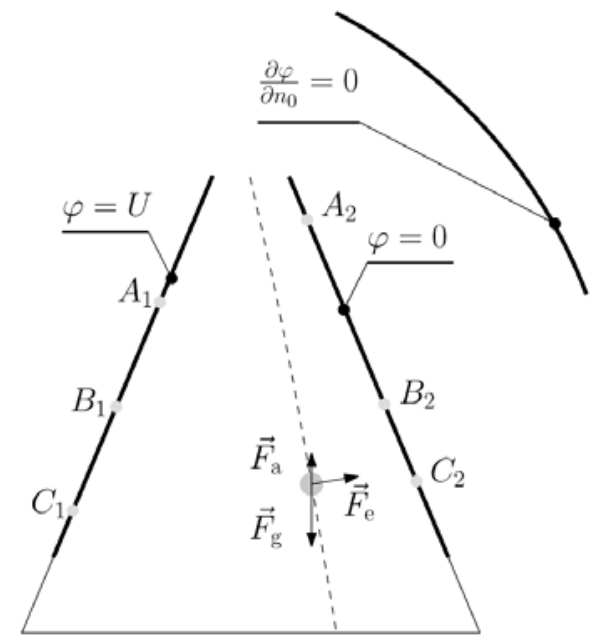

Fig. 7 Points $A_{1}, \ldots, C_{2}$ on the electrodes whose positions are to be optimized

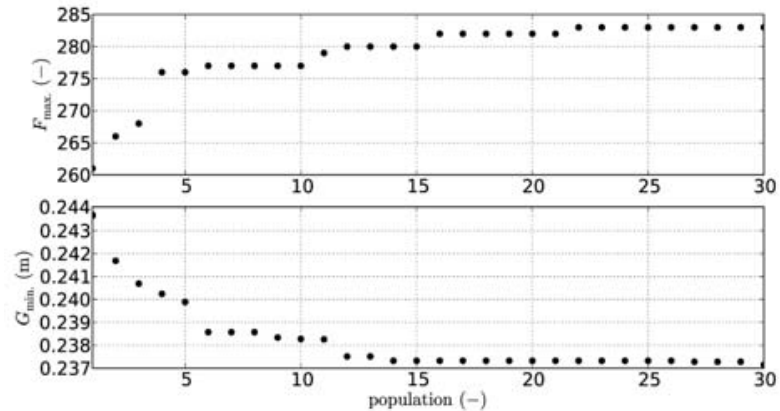

Fig. 8 Convergence of the functional $s F$ and $G$

Figure 9 shows particular populations and their influence on both functionals. A detail of the front part is shown in Fig. 10.

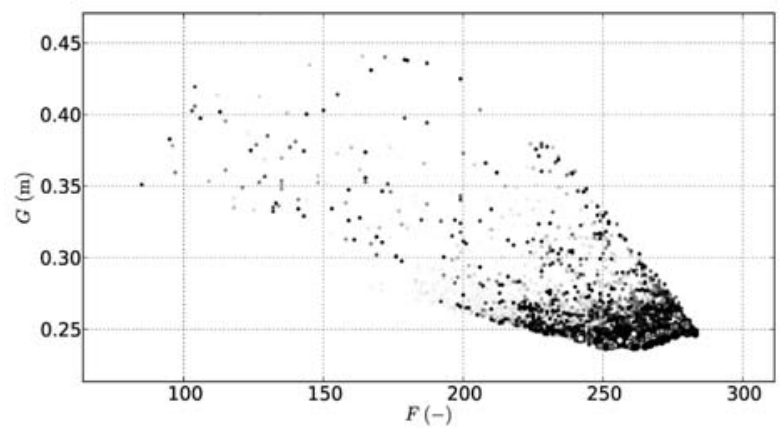

Fig. 9 Evolution of both functionals $F$ and $G$ with populations

The shape of the electrodes obtained after thirty populations is (for the variant of $F_{\max }$ ) depicted in Figs. 11 and 12 in common with trajectories of typical sets of the PET and PVC particles. In the entering part of the working chamber the particles are very close to both electrodes and the Coulomb forces acting on them reach the highest values. For the optimized variant, this fact is, moreover, supported by their shape. The vectors of electric force are here almost perpendicular to the trajectories of individual particles.

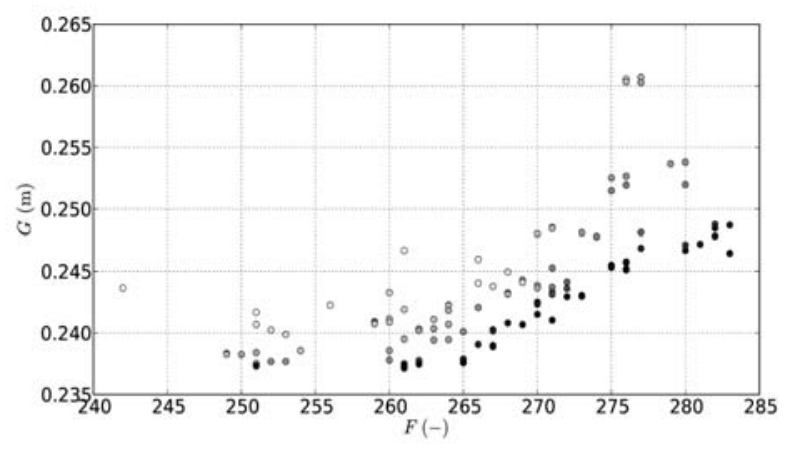

Fig. 10 Detail of the front part of Fig. 9 


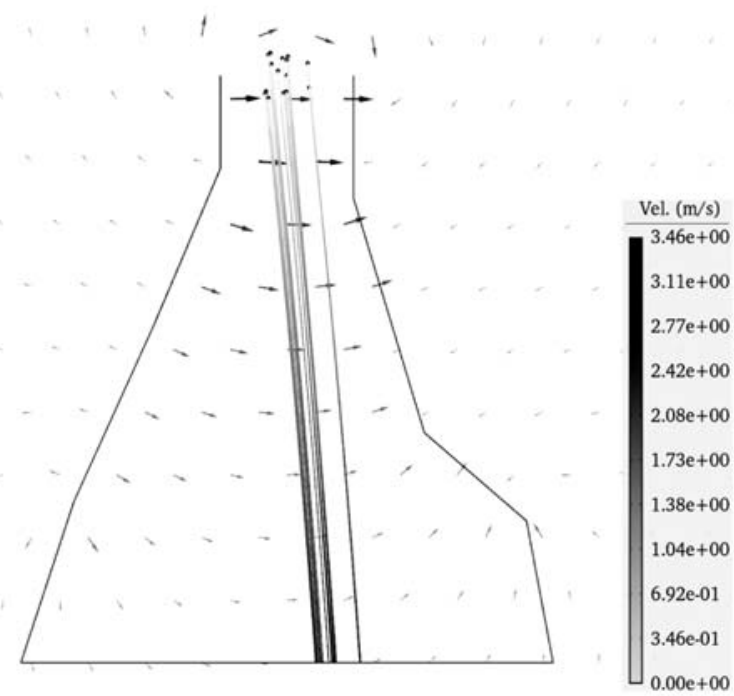

Fig. 11 Map of the trajectories and velocities of PET particles in the $30^{\text {th }}$ population

With respect to a considerable difference in the charge of both types of particles (the charge of the PVC particles is almost twice as high as the charge of the PET particles), the shapes of both electrodes differ substantially. The source electrode acting on the PVC particles is turned out of the working chamber. On the other hand, the grounded electrode is bent inward the working chamber. In this way, the change of the distance of both electrodes from the axis of the chamber leads to the balance of their forces on particular trajectories of the particles.

\section{Conclusion}

A triboelectric separator of plastic particles was modeled and optimized. First, we proposed a device with symmetrically arranged electrodes, mapped the distribution of electric field it and investigated the trajectories of the particular levels of plastics. The grains

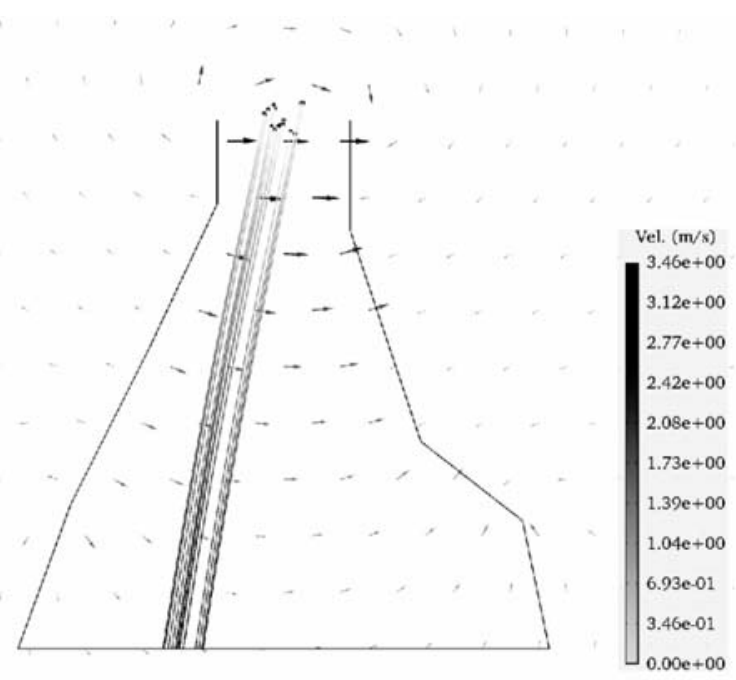

Fig. 12 Map of the trajectories and velocities of PVC particles in the $30^{\text {th }}$ population

of plastics were characterized by the normal distributions of both charges and dimensions. The efficiency of separation (evaluated from the viewpoint of the number of particles that fell down into the correct bins) was, however, low and did not exceed $47 \%$.

The second step was, therefore, to optimize the shapes of both electrodes in order to enlarge this quantity. After a thorough testing we suggested two objective functions to be extremized, which was realized by a suitable genetic algorithm. The modified shape of the electrodes leads to the increase of efficiency to $55.4 \%$.

\section{Acknowledgment}

This work was supported by the European Regional Development Fund and Ministry of Education, Youth and Sports of the Czech Republic (project No CZ.1.05/2.1.00/03.0094: Regional Innovation Centre for Electrical Engineering - RICE) and Grant project GACR P102/11/0498.

\section{References}

[1] SAEKI, M.: Triboelectric Separation of Three-Component Plastic Mixture, Particulate Science and Technology, vol. 26/5, pp. 494-506, 2008.

[2] MOESNER, F. M., TOSHIRO, H.: Contactless Manipulation of Microparts by Electric Field Traps. Proc. of SPIE's Intern. Symposium on Microrobotics and Microsystem Fabrication, Pittsburgh, vol. 3202, pp. 168-175, 1997.

[3] YANAR, D. K., KWETKUS, B. A.: Electrostatic Separation of Polymer Powders, J. of Electrostatics, vol. 36, No. 2-3, pp. 257-266, 1995.

[4] WEI, J., REALFF, M. J.: Design and Optimization of Free-Fall Electrostatic Separators for Plastics Recycling, AIChE Journal, vol. 49, No. 12, pp. 3138-3149, 2003.

[5] TILMATINE, A., MEDLES, K., YOUNES, M., BENDAOUD, A., DASCALESCU, L.: Roll-Type Versus Free-Fall Electrostatic Separation of Tribocharged Plastic Particles, IEEE Trans. Industry Appl., vol. 46, No. 4, pp. 1564-1569, 2010. 
[6] MACH, F., KUS, P., KARBAN, P., DOLEZEL, I.: Optimized Arrangement of Device for Electrostatic Separation of Plastic Particles. Proc. of conference ELEKTRO 2012, Zilina, 2012, CD-ROM.

[7] RUZICKOVA, M.: Discrete and Differential Equations in Applied Mathematics. Communications - Scientific Letters of the University of Zilina, No. 2, 2008.

[8] KUCZMANN, M., IVANYI, A.: The Finite Element Method in Magnetics, Akademiai Kiado, Budapest, 2008.

[9] Code Agros2D: http://agros2d.org.

[10] Code Hermes: http://hpfem.org/hermes. 